\title{
Changes in Protein Kinase A Activity Accompany Sclerotial Development in Sclerotinia sclerotiorum
}

\author{
A. Harel, R. Gorovits, and O. Yarden
}

Department of Plant Pathology and Microbiology and The Otto Warburg Minerva Center for Agricultural Biotechnology, Faculty of Agricultural, Food and Environmental Quality Sciences, The Hebrew University of Jerusalem, Rehovot 76100, Israel. Accepted for publication 15 December 2004.

\begin{abstract}
Harel, A., Gorovits, R., and Yarden, O. 2005. Changes in protein kinase A activity accompany sclerotial development in Sclerotinia sclerotiorum. Phytopathology 95:397-404.

Sclerotia of Sclerotinia sclerotiorum are pigmented, multihyphal structures that play a central role in the life and infection cycles of this pathogen. Sclerotial formation has been shown to be affected by increased intracellular cAMP levels. Cyclic AMP (cAMP) is a key modulator of cAMP-dependent protein kinase A (PKA) and the latter may prove to play a significant role in sclerotial development. Therefore, we monitored changes in relative PKA activity levels during sclerotial development. To do so, we first developed conditions for near-synchronous sclerotial development in culture, based on hyphal maceration and filter-

stage in the wild-type strain, while low levels were maintained in nonsclerotium-producing mutants. Furthermore, applying caffeine, an inducer of PKA activity, resulted in increased relative PKA activity levels and was correlated with the formation of sclerotial initial-like aggregates in cultures of the non-sclerotium-producing mutants. In addition, low PKA activities were found in an antisense $s m k 1$ strain, which exhibits low extracellular-signal-regulated kinase (ERK)-type mitogen-activated protein kinase (MAPK) activity, and does not produce sclerotia. The changes in PKA activity, as well as the abundance of phosphorylated MAPKs (ERK-like as well as p38-like) that accompany sclerotial development in a distinct developmental phase manner represent a potential target for antifungal intervention.
\end{abstract} ing. Relative PKA activity levels increased during the white-sclerotium
Additional keywords: oxalic acid.
Sclerotinia sclerotiorum is a necrotrophic, phytopathogenic, filamentous ascomycete. It is recognized as a broad-host-range, omnivorous plant pathogen with worldwide distribution. Over 400 species of plants are susceptible to this pathogen $(3,41,51)$. The sclerotium of $S$. sclerotiorum is a pigmented, asexual, multicellular, and firm resting structure composed of condensed vegetative hyphal cells which become interwoven and aggregate together and is capable of surviving years in soil (8). The sclerotium allows $S$. sclerotiorum to survive for long periods of time under adverse conditions. Sclerotia can remain dormant for 4 to 5 years in the soil and retain viability under variable conditions of humidity and temperature $(2,49,53)$. Sclerotia germinate myceliogenically to produce saprophytic or infectious hyphae, and carpogenically to form apothecia. Airborne ascospores derived from the apothecium are the most important means of $S$. sclerotiorum dispersal $(1,47)$.

Sclerotial development can be divided into three distinguishable stages (50): (i) initiation, the appearance of small distinct initial forms of interwoven hyphae, which develop terminally by repeated branching of long, aerial, primary hyphae and fusion of the branches (52); (ii) development, increase in size; and (iii) maturation, characterized by surface delimitation, internal consolidation, melanization, and, often, associated droplet secretion. These stages are accompanied by both morphological and biochemical differentiation. The initiation and maturation of sclerotia are affected by numerous factors such as photoperiod, temperature, oxygen concentration, mechanical factors, and nutrients (8).

Oxalic acid is known to play a role in S. sclerotiorum pathogenesis (20). Neutral or alkaline $\mathrm{pH}$ have been shown to increase

Corresponding author: O. Yarden; E-mail address: Oded.Yarden@huji.ac.il

DOI: 10.1094/PHYTO-95-0397

(C) 2005 The American Phytopathological Society oxalic acid accumulation and to inhibit sclerotial formation, whereas the process of oxalic acid accumulation leads to the lowering of ambient $\mathrm{pH}$ and forms conditions which favor sclerotial development (44). Thus, $\mathrm{pH}$-mediated signaling could function to regulate sclerotial development and oxalic acid production. The fact that oxalic acid-deficient mutants also have been shown to be unable to produce sclerotia is consistent with the importance of low medium $\mathrm{pH}$ in the process of sclerotial development (20).

Compounds known to increase endogenous cyclic AMP (cAMP) levels by inhibiting phosphodiesterase activity (caffeine and 3-isobutyl-1-methyl xanthine) or by activating adenylate cyclase $(\mathrm{NaF})$ reduce or eliminate sclerotial development in $S$. sclerotiorum. In addition, incorporation of cAMP into the growth medium decreases or eliminates the production of sclerotia and increases the accumulation of oxalic acid. Together, these results indicate that cAMP plays a role in the early transition between mycelial growth and sclerotial development (43). A prime candidate for mediating the influence of cAMP is the cAMPdependent protein kinase A (PKA) signal-transduction pathway.

PKA has been shown to mediate the regulation of different pathways in phytopathogenic fungi: dimorphic transition and pathogenicity in Ustilago maydis $(16,17)$, appressorium formation and pathogenesis in Magnaporthe grisea $(38,56)$, and pathogenesis in Colletotrichum trifolii $(58,59)$. The inactive form of PKA is composed of two catalytic subunits (C) and two regulatory subunits $(\mathrm{R})$. Binding of cAMP to two sites in each $\mathrm{R}$ subunit imposes a conformational change in these units, leading to the dissociation of the $\mathrm{R}_{2} \mathrm{C}_{2}$ complex into dimeric regulatory subunits and active, monomeric, catalytic subunits (39). The regulative role of PKA in the morphogenesis of phytopathogenic fungi and the demonstrated involvement of cAMP in sclerotial development and oxalic acid accumulation suggest that the cAMP effect may be mediated through PKA. It also is likely that, if a PKA pathway is involved, it functions in concert with additional path- 
ways, such as those involving small $\mathrm{G}$ proteins and mitogenactivated protein kinases (MAPKs).

MAPKs are involved in a variety of cellular pathways in pathogenic fungi. Among them are hyphal growth, virulence, appressorium formation or penetration, response to environmental stress (osmotic stress, UV), plant-fungus interactions, and pheromone signal transduction $(14,54,55)$. The first published genome sequence of a filamentous ascomycete (Neurospora crassa) indicated the presence of three MAPKs: MAK-2, OS-2, and a third MAPK, corresponding to Fus3/Kss1, HOG1, and Slt2/Mpk1 from Saccharomyces cerevisiae, respectively, in filamentous members of the fungal kingdom $(4,19)$. We have shown that the MAPK Smk1, an extracellular-signal-regulated kinase (ERK) cloned from Scelerotinia sclerotiorum, is essential for sclerotial development (7). In addition, smkl transcript levels were highest under acidic $\mathrm{pH}$, suggesting that Smk1 regulates sclerotial development through a $\mathrm{pH}$-dependent signaling pathway, involving the accumulation of oxalic acid. Because the addition of cAMP inhibited $s m k l$ transcription, MAPK activation, and sclerotial development, it is conceivable that Smk1 can serve as a mediator of environmental cues such as $\mathrm{pH}$ to induce sclerotial development in $S$. sclerotiorum, and that this pathway is negatively regulated by cAMP.

To date, the involvement of PKA in sclerotial development has not been demonstrated. Here, we report on the development of an inducible, near-synchronous, sclerotial development process in S. sclerotiorum and on the changes in PKA activity levels during sclerotial development. We found that relative PKA levels increase during sclerotial development in the wild-type strain, whereas low levels are maintained in non-sclerotium-producing mutants. Furthermore, applying environmental cues that elevate PKA activity was correlated with the formation of sclerotial initial-like aggregates in cultures of the non-sclerotium-producing mutants. We also found low PKA activity levels in the Smk1 mutant subjected to conditions that repress sclerotial development. Our results demonstrate clear fluctuations in the activity of PKA and the MAPKs, two of the backbone signal-transduction pathways in filamentous fungi.

\section{MATERIALS AND METHODS}

S. sclerotiorum growth conditions and synchronization of sclerotial formation. Wild-type $S$. sclerotiorum isolate 1980 and mutants A-1, A-3, and A-4 (20), as well as the Smk1 mutant (7), were used in this study. A-1, A-3, and A-4 are UV-induced prototrophic mutants deficient in oxalic acid production and sclerotial formation (20). The Smk1 strain harbors a construct expressing the $S$. sclerotiorum MAPK 1 gene $(s m k l)$ gene in an antisense orientation (7). Strains were routinely maintained on potato dextrose agar (Difco Laboratories, Detroit).

For all experimental procedures, unless otherwise stated, the fungus was grown on Joham's defined medium, $\mathrm{pH} 6.2$ (Joham) (26), consisting of glucose, $15 \mathrm{~g} ; \mathrm{MgSO}_{4}, 0.2 \mathrm{~g} ; \mathrm{K}_{2} \mathrm{HPO}_{4}, 0.6 \mathrm{~g}$; $\mathrm{KCl}, 0.15 \mathrm{~g} ; \mathrm{NH}_{4} \mathrm{NO}_{3}, 1 \mathrm{~g}$; thiamine chloride, $0.0001 \mathrm{~g}$; $\mathrm{FeSO}_{4}$, $0.009 \mathrm{~g} ; \mathrm{MnSO}_{4}, 0.008 \mathrm{~g} ; \mathrm{ZnSO}_{4}, 0.009 \mathrm{~g}$; and agar (when needed), $20 \mathrm{~g}$; per liter of medium. For the analysis of Smk1, the mutant was grown on modified Czapek-Dox liquid medium (Czapek-Dox) (42), consisting of $\mathrm{MgSO}_{4} 7 \mathrm{H}_{2} \mathrm{O}, 0.5 \mathrm{~g} ; \mathrm{K}_{2} \mathrm{HPO}_{4}$, $1 \mathrm{~g}$; KCL, $0.5 \mathrm{~g} ; \mathrm{NaNO}_{3}, 2 \mathrm{~g}$; and $\mathrm{FeSO}_{4} 7 \mathrm{H}_{2} \mathrm{O}, 0.01 \mathrm{~g}$; per liter of medium.

Synchronized sclerotial development was induced by plating blender-macerated and subsequently Büchner-filtered hyphal agar plugs on filter paper (Schleicher \& Schuell \#595) overlaying glass beads that were covered with liquid medium. Plates were incubated at $18^{\circ} \mathrm{C}$ and continuously shaken at $120 \mathrm{rpm}$. In this system, part of the mycelium (adjacent to the filter paper) was in contact with the medium while the other was exposed to air, which induced sclerotial formation.
Western blot analysis. Following different treatments, cultures were harvested and immediately frozen in liquid nitrogen, ground, and suspended in extraction buffer consisting of $1 \mathrm{M}$ Sorbitol, $10 \mathrm{mM}$ HEPES (pH 7.5), 5 mM EDTA, 5 mM EGTA, 5 mM NaF, $0.1 \mathrm{M} \mathrm{KCl}, 0.2 \%$ (wt/vol) Triton X-100, and Complet (Roche Applied Science, Mannheim, Germany) protease inhibitor mixture. After 40 to $45 \mathrm{~min}$ of incubation on ice, samples were centrifuged for $40 \mathrm{~min}$ at $10,000 \times g$ and the supernatant was transferred to a new tube. Cell-lysate protein concentrations were quantified by the Bradford protein assay (5). Proteins were resolved by 10 or $12 \%$ (wt/vol) sodium dodecyl sulfate polyacrylamide gel electrophoresis (SDS-PAGE). Western blotting was performed by standard procedures (46). Antibodies used throughout this study included anti-phospho-MAPK antibodies raised against mammalian ERK1/2 (MAPK/extracellular signal-regulated kinases) and p38 (New England Biolabs, Beverly, MA) and goat peroxidase-coupled secondary antibodies (Sigma-Aldrich, St. Louis).

PKA activity assays. Protein extracts prepared from $S$. sclerotiorum cultures subjected to different treatments were first frozen in liquid nitrogen and then ground (twice, for $30 \mathrm{~s}$, at 4,000 rpm) with 0.5 -mm glass beads in a Bio101 bead beater (Savant, Farmingdale, NY) in extraction buffer consisting of $50 \mathrm{mM}$ Tris- $\mathrm{HCl}$ (pH 7.5), $5 \mathrm{mM}$ EDTA, $5 \mathrm{mM}$ EGTA, $10 \mathrm{mM}$ $\mathrm{NaF}, 0.1 \mathrm{M} \mathrm{KCl}, 0.5 \%$ (vol/vol) Triton X-100, and $0.2 \%$ (wt/vol) SDS. The homogenate was centrifuged for $40 \mathrm{~min}$ at $10,000 \times g$ and the supernatant recovered for PKA activity assays. Equal quantities (as determined by Bradford protein assay) of protein extract were utilized for further analysis of PKA activity.

Relative in vitro PKA activity was determined on the basis of kemptide phosphorylation, as measured with the PepTag kit (Promega Corp., Madison, WI) and with minor modifications of the manufacturer's instructions (the PKA activator was diluted 10fold and the enzymatic reaction was carried out for $60 \mathrm{~min}$ ). The PepTag assays utilize fluorescent peptide substrates specific for PKA. Phosphorylation of the substrate by PKA alters the peptide's net charge from +1 to -1 , allowing separation of the phosphorylated substrate from the nonphosphorylated one on an $1.5 \%$ agarose gel, $50 \mathrm{mM}$ Tris- $\mathrm{HCl}(\mathrm{pH} \mathrm{7.5)}$. All experiments testing for PKA activity were repeated three times.

\section{RESULTS}

Synchronization of sclerotial development. Under standard culturing conditions, sclerotial production is not synchronous and thus limits the possibility of following the kinetics of biochemical reactions or gene expression. Therefore, we developed a method for obtaining nearly synchronized production of sclerotia. On day 2 after incubation, hyphal aggregates appeared, indicating the formation of sclerotium initials that many of them eventually developed into sclerotia. On day 3, the sclerotia initials developed to form white rigid structures often accompanied by droplet secretion. By day 4, melanization of the rigid sclerotial initials and formation of melanized sclerotia was evident (Fig. 1A). Under standard growth conditions (e.g., when petri dishes containing solid medium are inoculated, in their center, with mycelial discs and incubated at $18^{\circ} \mathrm{C}$ ), sclerotial development accompanies the progression of radial hyphal growth. Typically, sclerotial initials appear after 3 to 4 days and their number gradually accumulates over the culturing period. In contrast, after maceration and exposure to air, most of the sclerotial initials appear 3 days after induction. Thus, even though the number of sclerotial initials in standard and synchronized cultures is similar, their rate of accumulation in near-synchronized cultures is markedly steeper and most sclerotium initials are already visible after the third day (Fig. 1B). Therefore, our protocol provides a convenient tool for studying the characteristics of sclerotial development and was used throughout this study. 
Alterations in PKA activity during sclerotial development. To analyze the possible involvement of PKA during sclerotial development, we measured relative in vitro PKA activity in extracts obtained from different morphological stages of sclerotial development (initials, white sclerotia, and melanized sclerotia). Clear differences in relative PKA activity were observed during the different phases of sclerotial development in cultures induced to produce sclerotia. Whereas only minimal activity was detected during the initiation stage, significant increases were observed during the formation of white sclerotia, followed by a reduction in PKA activity, as detected in the melanized sclerotial extracts (Fig. 2A).

Additional evidence for the possible involvement of PKA in sclerotial development could be deduced by measuring relative PKA activity in $S$. sclerotiorum mutants (A-1, A-3, and A-4) that failed to produce sclerotia (20). Using the described protocol, PKA activity was determined to be low in the non-sclerotiumproducing strains of $S$. sclerotiorum, when compared, in parallel, with that of the white and melanized sclerotial stages in the wildtype cultures induced to form sclerotia. These lower PKA-activity levels were consistent in all three mutants tested (Fig 2B).

These results are consistent with previous reports demonstrating that PKA mediates the regulation of various morphological pathways in other phytopathogenic fungi $(16,17,38,56,58,59)$. It is likely that at least some of the effects of PKA can be directed or mediated through additional signal-transduction pathways. A prime candidate for such an additional pathway is the MAPK pathway (33).

Induction of PKA activity is correlated with production of sclerotial initial-like aggregates in non-sclerotium-producing mutants of $S$. sclerotiorum. Caffeine has been shown to increase the accumulation of endogenous cAMP levels in S. sclerotiorum (43). This mechanism is inferred to be inhibiting phosphodiesterase activity. Because PKA is activated through binding to cAMP
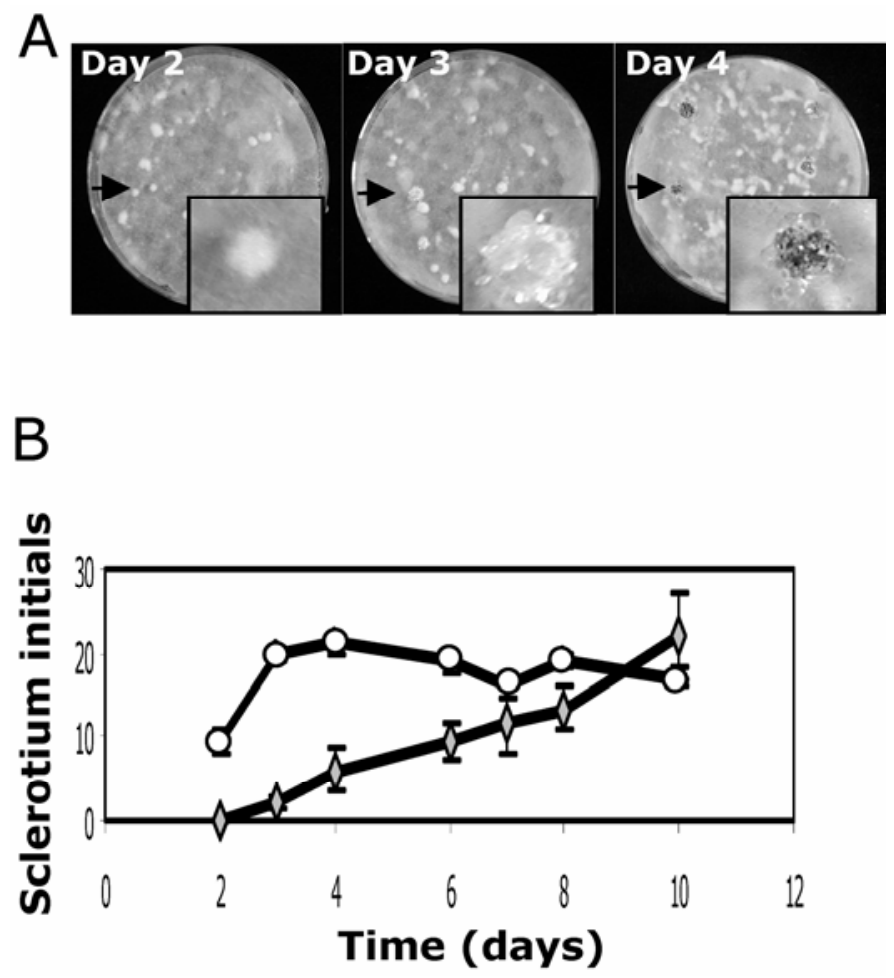

Fig. 1. A, Near-synchronous sclerotial development following maceration and filtration of hyphal cultures. Sclerotium initials, white sclerotia, and melanized sclerotia are visible after 2, 3, and 4 days, respectively. Magnified examples of typical developmental stages are shown (arrows). B, Accumulation of sclerotium initials in standard and near-synchronous cultures (diamonds and circles, respectively). Bars indicate standard error.
(39), it is reasonable to postulate that the addition of caffeine to the $S$. sclerotiorum culture media will lead to an increase in PKA activity by elevating the endogenous level of cAMP within the fungal cell. PKA activity was correlated with sclerotial development in the wild type, and it was low in the non-sclerotium-producing mutants; therefore, the influence of caffeine, as an inducer of PKA activity, on the morphology of the non-sclerotium-producing mutants was tested.

Inoculating macerated hyphae of A-1, A-3, and A-4 mutants on media containing $2.5 \mathrm{mM}$ caffeine resulted in the production of morphological structures consisting of condensed hyphae that visually resembled the sclerotial initials formed in the wild type (Fig. 3A). These initial-like aggregates did not proceed to produce mature sclerotia in time or in higher concentrations of caffeine. This phenomenon was evident in the three mutants examined. In contrast, treating the mutant strain-hyphae with $5 \mu \mathrm{M}$ Polyoxin $\mathrm{D}$, a chitin-synthase inhibitor, did not invoke this morphological effect, suggesting that imposing an unrelated stress does not confer this phenotypic alteration. Because the presence of caffeine confers cAMP accumulation, we tested whether caffeine induces an increase in PKA activity in the sclerotial initial-like aggregates. Indeed, the appearance of sclerotial initial-like aggregates, induced by caffeine, in the non-sclerotium-producing mutants was correlated with increased levels of PKA activity (Fig. 3B).

\section{A}
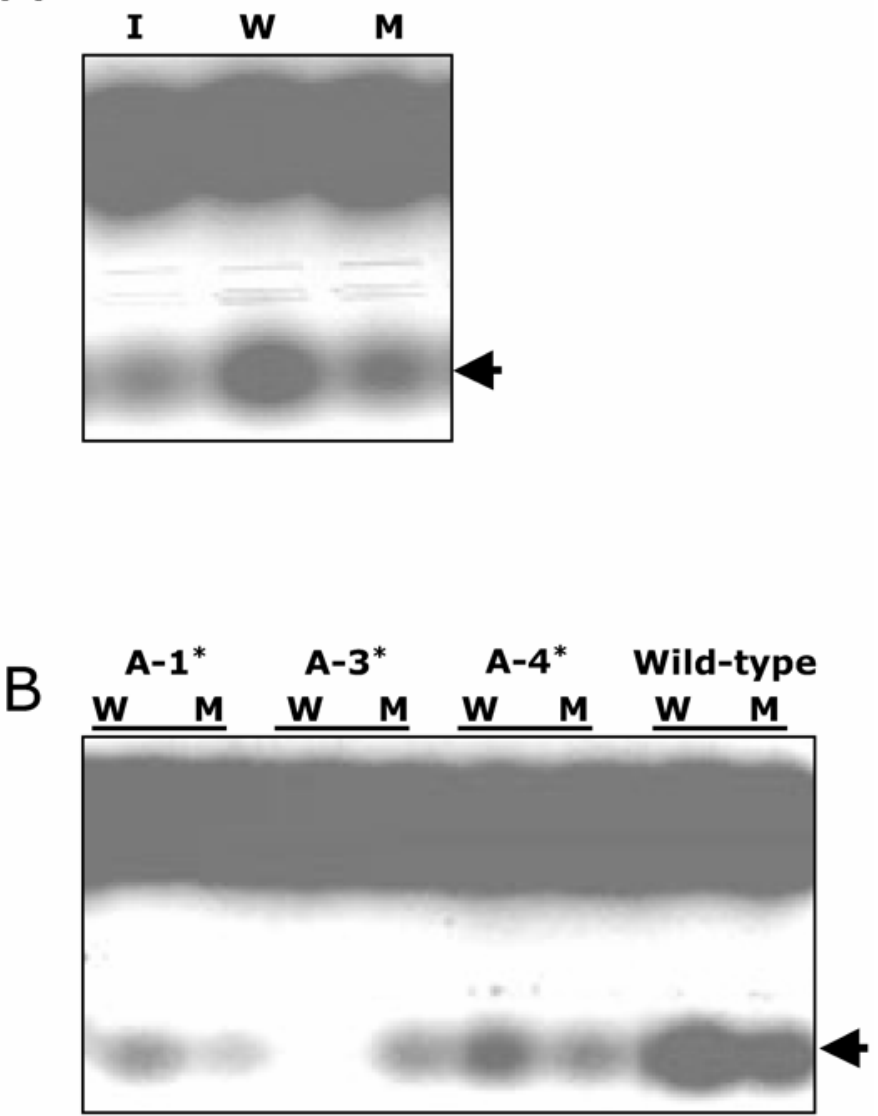

Fig. 2. A, Relative in vitro protein kinase A (PKA) activity, as measured in protein extracts prepared from different developmental stages of sclerotial development after synchronization in Sclerotinia sclerotiorum cultures ( $\mathrm{I}=$ initials at $48 \mathrm{~h}, \mathrm{~W}=$ white at $72 \mathrm{~h}$, and $\mathrm{M}=$ melanized sclerotia at $96 \mathrm{~h}$ ). The phosphorylated (indicating activity) fluorescent kemptide substrate is marked with an arrow. B, PKA activity in the wild-type and non-sclerotia producing strains of $S$. sclerotiorum at different time points after synchronization for sclerotial development. The phosphorylated (indicating activity) fluorescent kemptide substrate is marked with an arrow. In strains that do not produce sclerotia (marked by an asterisk), W and M signify 72 or $96 \mathrm{~h}$ postinduction, respectively. 
Sodium succinate induces sclerotial initial-like aggregate development in non-sclerotium-producing mutants of $S$. sclerotiorum. Addition of sodium succinate, an inducer of oxalic acid production (37), to the A-1, A-3, and A-4 mutants induced accumulation of oxalic acid in the culture media and reestablished pathogenesis (20). When testing for morphological changes, we found that, after plating macerated A-3 strain hyphae on medium containing $1.5 \%$ (wt/vol) sodium succinate, hyphal aggregates resembling sclerotial initials formed. This effect was significantly enhanced when the growth medium also was amended with $2.5 \mathrm{mM}$ caffeine (Fig. 4). The same phenomenon was observed for A-1 and A-4 non-sclerotium-producing mutants. Moreover, in additional experiments, we determined that the presence of sodium succinate does not inhibit hyphal growth. Thus, we concluded that the morphological changes observed in the mutants are not the result of an inhibitory effect of sodium succinate on

A

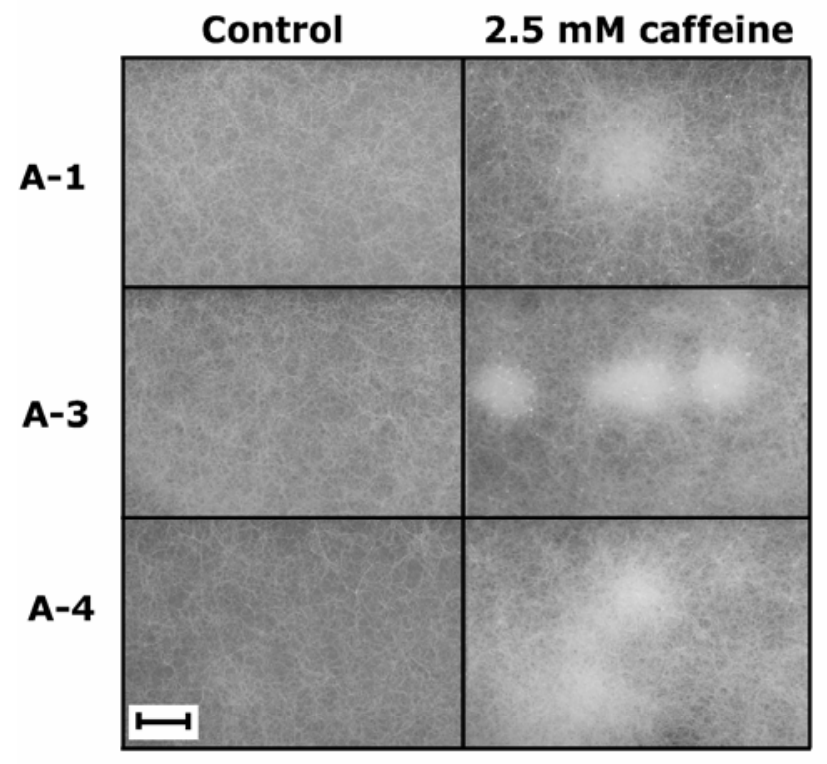

B

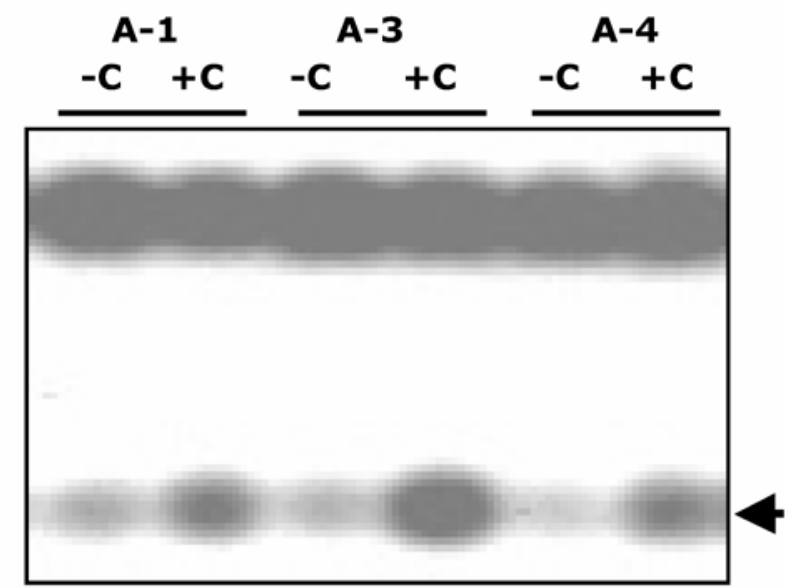

Fig. 3. A, Effect of caffeine on non-sclerotium-producing mutant (A-1, A-3, and A-4) morphology. Mutant hyphae were induced for sclerotial development and plated on Joham media either unamended (control), or amended with caffeine $(2.5 \mathrm{mM})$. Bar indicates $2 \mathrm{~mm}$. B, Relative in vitro protein kinase A activity as measured in protein extracts prepared from non-sclerotiumproducing mutants (A-1, A-3, and A-4) induced for sclerotia production and plated on Joham medium unamended $(-\mathrm{C})$, or amended $(+\mathrm{C})$ with caffeine (2.5 mM).

hyphal elongation. To determine whether sodium succinate-mediated sclerotial development is correlated with increased PKA activity, we examined whether the appearance of hyphal aggregates in the non-sclerotium-producing mutants in the presence of sodium succinate was accompanied by an increase in PKA activity levels. PKA activity levels of the three mutants grown in the presence of sodium succinate was not altered relative to growth on control media, consistent with the possible presence of a PKAindependent pathway (C. Chen and M. B. Dickman, unpublished data) regulating sclerotium formation.

Alteration of phosphorylated MAPK protein levels during sclerotial development. MAPKs are involved in a variety of cellular pathways that regulate morphogenesis in pathogenic fungi $(14,54,55)$. Previously, we demonstrated that Smk1, an ERK MAPK of S. sclerotiorum, is essential for sclerotial development (7). Here, we expanded the analysis of MAPK protein phosphorylation during different stages of sclerotial development. The levels of active ERK1/2 (corresponding to Smk1 in S. sclerotiorum) and p38 (corresponding to HOG1 in S. cerevisiae) were detected with polyclonal antibodies against the phosphorylated, active forms of these proteins (Fig. 5). We found an increase in

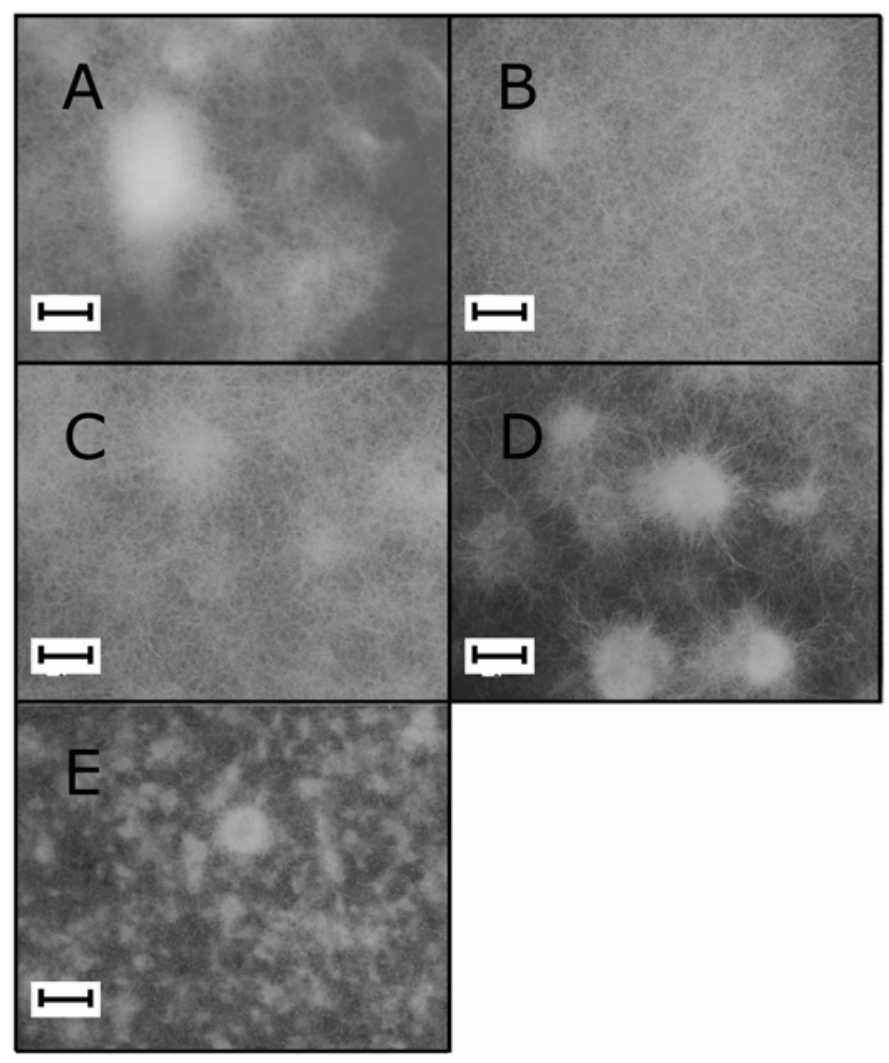

Fig. 4. Effect of different treatments on the induction of hyphal aggregates formation in the A-3 mutant. A, Sclerotial initials of the wild-type strain, B, A-3 on unamended medium, C, A-3 grown in the presence of $2.5 \mathrm{mM}$ caffeine, D, A-3 grown in the presence of $1.5 \mathrm{mM}$ sodium succinate, and E, A-3 grown in the presence of $2.5 \mathrm{mM}$ caffeine and $1.5 \%$ (wt/vol) sodium succinate. Bars indicate $2 \mathrm{~mm}$.

A

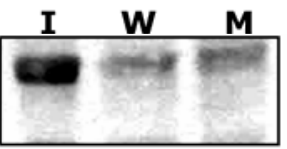

Fig. 5. Western analysis of Sclerotinia sclerotiorum protein extracts probed with $\mathbf{A}$, anti-38-Ph (homologous to HOG1) antibodies or B, anti-P42/44-Ph (ERK) antibodies $(\mathrm{I}=$ initials, $\mathrm{W}=$ white, and $\mathrm{M}=$ melanized sclerotia $)$. 
the phosphorylated forms of ERK and p38 during the stage of sclerotial initiation, which than decreased as the sclerotia matured, demonstrating the likely involvement of more than one MAPK, functioning simultaneously, in S. sclerotiorum sclerotial development. These results were consistent in protein extracts obtained from three independent experiments in which cultures had been induced to form sclerotia.

Relative PKA activity during sclerotial development in an Smk1 mutant. There is growing evidence of cross-talk between PKA and MAPK pathways in fungal pathogens $(28,32,34)$ and in Saccharomyces cerevisiae (45). Furthermore, in several cases, studies in mammalian systems have suggested that the PKA pathway regulates MAPK pathways $(10,11,24,57)$. To examine the potential link between MAPK and PKA in Sclerotinia sclerotiorum, we utilized a $S$. sclerotiorum transformant, in which an inducible antisense RNA is used to regulate Smk1 expression (7). Relative activity of PKA was measured in protein extracts prepared from smkl mutant hyphae grown under conditions in which smkl antisense expression was induced (15 mM quinic acid) or repressed (15 mM glucose). The levels of PKA activity were lower in the antisense smkl strain grown in the presence of quinic acid in contrast to increased PKA activity levels measured in the antisense $s m k 1$ strain grown on medium supplemented with glucose (Fig. 6A). The presence of quinic acid in the culture medium of the wild-type strain did not reduce PKA activity (Fig. $6 \mathrm{~B}$ ), indicating that the changes in PKA activity are not a result of an alternative effect induced by the presence of quinic acid in the culture medium. Thus, as with the reduced PKA levels observed in the A-1, A-3, and A-4 mutants, a similar phenomenon could be observed in a genetically characterized mutant that is impaired in sclerotial production.

\section{DISCUSSION}

S. sclerotiorum is capable of survival for years in soil (8) and spends approximately $90 \%$ of its life cycle as sclerotia. Airborne ascospores, derived from apothecia which develop from the sclerotia, are the most important means of $S$. sclerotiorum dispersal $(1,47)$. Therefore, sclerotia are a major means of survival and, in many instances, serve as the source of initial inoculum of plant diseases caused by $S$. sclerotiorum $(1,2,47$, 49,53). Understanding the physiological and molecular mechanisms involved in sclerotial development in S. sclerotiorum can enhance our understanding of sclerotial development in various fungi and may provide new avenues for intervention in this process, leading to improved control of disease caused by sclerotium-borne fungi.

Synchronization of sclerotial growth. A protocol for the induction of nearly synchronous sclerotial development was established. In the basidiomycete Sclerotium rolfsii, sclerotium formation on agar plates usually occurs when the mycelium approaches the edge of the petri dish. However, the process also can be induced by shearing the mycelium (23); amending the growth medium with iodoacetic acid (9), lactose (40), or threonine (30); or transferring the mycelium from a submerged to an aerial growth environment (22). Not all the treatments inducing sclerotial development in $S$. rolfsii affect this process in Sclerotinia sclerotiorum. For example, addition of threonine or iodoacetic acid to the culture medium of $S$. sclerotiorum did not result in synchronous induction of sclerotial development (O. Erner and $\mathrm{O}$. Yarden, unpublished data). However, combining two of the treatments shown to induce sclerotial formation in Sclerotium rolfsii (i.e., shearing of the mycelium [23] and switching it from submerged to aerial growth [22]) resulted in the near-synchronous formation of sclerotia in Sclerotinia sclerotiorum. In this study, our protocol for inducing nearly synchronous sclerotial development enabled the characterization of changes in PKA and MAPK activity during sclerotial development. This tool may prove useful in future analyses of sclerotial development in S. sclerotiorum and other fungi.

The involvement of PKA during sclerotial development. The importance of PKA in the regulation of morphogenesis in other fungi $(16,17,38,56)$ led us to investigate the possible involvement of this enzyme in sclerotial development. The increase in relative PKA activity levels during the production of white sclerotia implied that PKA activity is most likely involved in sclerotial development.

This possibility was further supported by the observation that, in non-sclerotium-producing mutants, PKA activity levels were much lower at time points paralleling the appearance of white sclerotia or melanized sclerotia in the wild type. To further test our hypothesis, we subjected the non-sclerotium-producing mutants exhibiting low PKA activity to caffeine, which is known to induce an increase in PKA activity. As expected, this treatment did increase PKA activity (Fig. 3B). Interestingly, this increase in relative enzyme activity also was accompanied by the formation of sclerotial initial-like aggregates. We propose that the caffeineinduced increase in PKA activity was due to an increase in intracellular cAMP levels (43), even though caffeine has been shown to be capable of exerting its activity via additional pathways $(35,36)$. The observation that caffeine induces hyphal fusion in the filamentous ascomycete $N$. crassa (A. Harel and O. Yarden, unpublished data) raises the intriguing possibility that this also may be the case in $S$. sclerotiorum, because anastomosis was shown to play a role during the initiation stage of sclerotial development in this pathogen (52).

Further support of the hypothesis that PKA is involved in sclerotial development can be deduced from the observation that an smkl antisense strain, an additional strain that does not produce sclerotia, exhibited low levels of PKA activity when grown in conditions that induce smkl antisense expression $(15 \mathrm{mM}$ quinic acid).

A PKA catalytic subunit gene recently was cloned and disrupted in S. sclerotiorum (27). Disrupted strains still produced sclerotia and were cAMP responsive. However, significant levels of PKA activity were still present, suggesting the existence of a second PKA catalytic subunit functioning in this fungus. C. Chen and M. B. Dickman (unpublished data) have demonstrated that

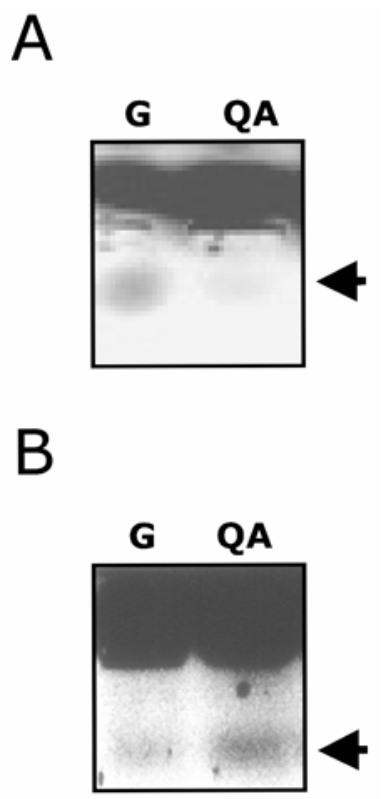

Fig. 6. A, Protein kinase A (PKA) activity in hyphal extracts of the SMK1 strain, grown in the presence of $15 \mathrm{mM}$ glucose $(\mathrm{G}$, antisense repressor) or $15 \mathrm{mM}$ quinic acid (QA, antisense inducer). B, PKA activity in hyphal extracts of the wild-type strain grown in the presence of $15 \mathrm{mM} \mathrm{G}$ or $15 \mathrm{mM}$ QA. 
the expression of a dominant negative form of S. sclerotiorum Ras also inhibits sclerotial development and MAPK activation. In $S$. cerevisiae Ras, proteins are known to interact with the enzyme adenylyl cyclase and stimulate the production of cAMP $(18,48)$ which, in turn, results in the activation of PKA to induce the Ras/PKA pathway (6). Our current results, demonstrating the developmentally coordinated changes in PKA activity, together with the evidence for an Ras/MAP pathway, emphasize the requirement to further determine the hierarchical nature of PKA's involvement in sclerotial development.

In summary, we established four lines of evidence for the specific involvement of PKA in sclerotial development: (i) the correlation between changes in relative PKA activity and sclerotial development in the wild-type strain; (ii) the low relative PKA activity observed in non-sclerotium-producing mutants; (iii) morphological changes (sclerotial initial-like aggregate formation) in nonsclerotium-producing mutants induced by caffeine, a PKA activity activator, which correlated with an accompanying increase in PKA levels; and (iv) low PKA activity in the smkl mutant subjected to conditions that prevented sclerotial development.

Additional evidence for the involvement of PKA in sclerotial development potentially could be produced by the use of specific PKA inhibitors, such as H89 or KT5720. However, these compounds do not affect sclerotial development in vivo (43). This is not the only case of significant differences between in vivo and in vitro activity of inhibitors tested in fungi. For example, it has been shown that okadaic acid, a specific inhibitor of protein phosphatase type 2A and 1 (PP2A and PP1), does not inhibit hyphal growth of $N$. crassa, even though it is a potent inhibitor of $N$. crassa PP2A in vitro (31). This reemphasizes the potential limitations of using enzyme inhibitors, which are otherwise useful tools in dissecting cellular processes.

Sodium succinate mediated sclerotial initial-like aggregate formation. Oxalic acid accumulation is involved in sclerotial development (44) and the addition of sodium succinate, an inducer of oxalic acid production (37), to the A-1, A-3, and A-4 mutants induced accumulation of oxalic acid in the culture media (20); therefore, we determined whether sodium succinate could invoke sclerotial development in the non-sclerotium-producing mutants. Amending the standard growth medium with sodium succinate had no effect on sclerotial development in the mutants. However, sclerotial initial-like aggregates were evident when sodium succinate was amended to the media in which macerated hyphae had been grown. These results imply that the effect of sodium succinate is exerted at a stage downstream of the effect induced by maceration and exposure to air and cannot compensate for the cellular processes triggered by the physical treatment. Induction of sclerotial initial-like-aggregate forms in the non-sclerotiumproducing mutants by sodium succinate was not accompanied by an increase in PKA activity levels. This result also could imply that this pathway is downstream of PKA, or that there is an additional PKA-independent pathway involving succinate or oxalate which mediates sclerotial development. Determining the genetic defect in the non-sclerotium-producing strains could contribute significantly to our understanding of the process involved, but attempts to clone the relevant genes have been unsuccessful (M. B. Dickman, unpublished data).

The involvement of MAPKs in sclerotial development of S. sclerotiorum. We previously showed that Smk1 is an essential MAPK for sclerotial development during the formation of sclerotium initials (7). We explored whether this phenomenon is related to more than one MAPK species. The observation that the activity of the $S$. sclerotiorum HOG1 homologue also is correlated with sclerotial development and is coordinated with changes in Smk1 phosphorylation implies that sclerotial development is a complex process in which more than one MAPK is involved. HOG1 and its homologues are involved in regulating the osmoticstress response in $S$. cerevisiae and other fungi $(13,15,21,54,60)$.
Sclerotial development is accompanied by the accumulation of carbohydrates capable of inducing osmotic stress, such as trehalose and mannitol (53); therefore, sclerotial development may be accompanied by an increase in intracellular osmolyte accumulation. Thus, an increase in the levels of active HOG1 could mediate an osmotic-stress response which may accompany sclerotial development.

Recently, research in $U$. maydis has presented evidence of crosstalk between the cAMP-PKA signaling pathway and a MAPK pathway influenced by Ras2 (33). Ras2-suppressor mutations were identified that restored budding growth to a constitutively filamentous mutant with a defect in the gene encoding a catalytic subunit of PKA. Whether or not a similar form of crosstalk functions in $S$. sclerotiorum has yet to be determined.

Interconnecting pathways of CAMP and PKA for sclerotial development. Spatial and temporal regulation of the activity of signal-transduction components is a prerequisite for their successful function. Thus, alterations in PKA or MAPK activity may have a significant influence on the outcome of the processes they regulate. The development of the complex morphological sclerotium of $S$. sclerotium is a multistage process which is activated by genetically based developmental programs and influenced by environmental changes. Some of these processes are mediated by signal-transduction pathways, which we detected here by monitoring the changes in activity of biochemical components such as PKA and MAPK.

cAMP - an inducer of PKA activity - has been shown to negatively affect sclerotial development (43). This may appear to contradict our findings that increased PKA activity accompanies sclerotial development and that induction of PKA activity can enhance the initial phase of sclerotial development in non-sclerotium-producing mutants. However, during sclerotial development, PKA activity levels change dramatically with time. Therefore, it may be that the temporal or quantitative aspects of PKA activity levels are significant components of PKA-mediated morphology in S. sclerotiorum. Thus, constant PKA activity (as may be imposed by the presence of cAMP in the growth medium) may result in the inhibition of sclerotial development during the early phase, in line with the apparent requirement for low PKA activity levels that we found in hyphal initials, which may be part of the process regulating the initiation of the morphological process. Alternatively, some of the effects imposed by increased intracellular cAMP levels may not be mediated by PKA. Though such non-PKA mediators have yet to be found in S. sclerotiorum, proteins known to interact with cAMP have been detected in other organisms $(12,25,29,39)$.

Functional and sequence analyses have demonstrated the existence of more than one PKA catalytic subunit in several fungi $(4,17,19,27,56)$. Therefore, it is conceivable that this may be the case in $S$. sclerotiorum as well. Isolation and subsequent inactivation (or overexpression) of the related genes (single or double disruption) will help determine the degree of PKA involvement in sclerotial development and its possible interaction with the signal-transduction pathways in regulating morphogenesis in S. sclerotiorum.

\section{ACKNOWLEDGMENTS}

This research was supported by BARD, the United States-Israel Binational Agricultural Research and Development Fund.

\section{LITERATURE CITED}

1. Abawi, G. S., and Grogan, G. 1979. Epidemiology of diseases caused by Sclerotinia species. Phytopathology 69:899-904.

2. Adams, P. B., and Ayers, W. A. 1979. Ecology of Sclerotinia species. Phytopathology 69:896-899.

3. Boland, G. J., and Hall, R. 1994. Index of plant hosts of Sclerotinia sclerotiorum. Can. J. Plant Pathol. 16:93-108. 
4. Borkovich, K. A., Alex, L. A., Yarden, O., Freitag, M., Turner, G. E., Read, N. D., Seiler, S., Bell-Pedersen, D., Paietta, J., Plesofsky, N., Plamann, M., Goodrich-Tanrikulu, M., Schulte, U., Mannhaupt, G., Nargang, F. E., Radford, A., Selitrennikoff, C., Galagan, J. E., Dunlap, J. C., Loros, J. J., Catcheside, D., Inoue, H., Aramayo, R., Polymenis, M., Selker, E. U., Sachs, M. S., Marzluf, G. A., Paulsen, I., Davis, R., Ebbole, D. J., Zelter, A., Kalkman, E. R., O'Rourke, R., Bowring, F., Yeadon, J., Ishii, C., Suzuki, K., Sakai, W., and Pratt, R. 2004. Lessons from the genome sequence of Neurospora crassa: Tracing the path from genomic blueprint to multicellular organism. Microbiol. Mol. Biol. Rev. 68:1-108.

5. Bradford, M. M. 1976. A rapid and sensitive method for the quantitation of microgram quantities of protein utilizing the principle of protein-dye binding. Anal. Biochem. 72:248-254.

6. Chang, Y. W., Howard, S. C., and Herman, P. K. 2004. The Ras/PKA signaling pathway directly targets the Srb9 protein, a component of the general RNA polymerase II transcription apparatus. Mol. Cell 15:107-116.

7. Chen, C., Harel, A., Gorovoits, R., Yarden, O., and Dickman, M. B. 2004. MAPK regulation of sclerotial development in Sclerotinia sclerotiorum is linked with $\mathrm{pH}$ and cAMP sensing. Mol. Plant-Microbe Interact. 17:404413.

8. Chet, I., and Henis, Y. 1975. Sclerotial morphogenesis in fungi. Annu. Rev. Phytopathol. 13:169-192.

9. Chet, I., Henis, I., and Mitchell, R. 1966. The morphogenetic effect of sulphur-containing amino acids, glutathione and iodoacetic acid on Sclerotium rolfsii Sacc. J. Gen. Microbiol. 45:541-546.

10. Chio, C. C., Chang, Y. H., Hsu, Y. W., Chi, K. H., and Lin, W. W. 2004. PKA-dependent activation of PKC, p38 MAPK and IKK in macrophage: Implication in the induction of inducible nitric oxide synthase and interleukin-6 by dibutyryl cAMP. Cell Signal. 16:565-575.

11. Crepieux, P., Martinat, N., Marion, S., Guillou, F., and Reiter, E. 2002. Cellular adhesion of primary Sertoli cells affects responsiveness of the extracellular signal-regulated kinases 1 and 2 to follicle-stimulating hormone but not to epidermal growth factor. Arch. Biochem. Biophys. 399:245-250.

12. Cui, J., Kagan, A., Qin, D., Mathew, J., Melman, Y. F., and McDonald, T. V. 2001. Analysis of the cyclic nucleotide binding domain of the HERG potassium channel and interactions with KCNE2. J. Biol. Chem. 276:17244-17251.

13. De Nadal, E., Zapater, M., Alepuz, P. M., Sumoy, L., Mas, G., and Posas, F. 2004. The MAPK HOG1 recruits Rpd3 histone deacetylase to activate osmoresponsive genes. Nature 427:370-374

14. Dickman, M. B., and Yarden, O. 1999. Serine/threonine protein kinases and phosphatases in filamentous fungi. Fungal Genet. Biol. 26:99-117.

15. Dixon, K. P., Xu, J. R., Smirnoff, N., and Talbot, N. J. 1999. Independent signaling pathways regulate cellular turgor during hyperosmotic stress and appressorium-mediated plant infection by Magnaporthe grisea. Plant Cell 11:2045-2058.

16. D'Souza, C. A., and Heitman, J. 2001. Conserved cAMP signaling cascades regulate fungal development and virulence. FEMS Microbiol. Rev. 25:349-364.

17. Durrenberger, F., Wong, K., and Kronstad, J. W. 1998. Identification of a cAMP-dependent protein kinase catalytic subunit required for virulence and morphogenesis in Ustilago maydis. Proc. Natl. Acad. Sci. USA 95:5684-5689.

18. Field, J., Xu, H. P., Michaeli, T., Ballester, R., Sass, P., Wigler, M., and Colicelli, J. 1990. Mutations of the adenylyl cyclase gene that block RAS function in Saccharomyces cerevisiae. Science 247:464-467.

19. Galagan, J. E., Calvo, S. E., Borkovich, K. A., Selker, E. U., Read, N. D., Jaffe, D., FitzHugh, W., Ma, L. J., Smirnov, S., Purcell, S., Rehman, B., Elkins, T., Engels, R., Wang, S., Nielsen, C. B., Butler, J., Endrizzi, M., Qui, D., Ianakiev, P., Bell-Pedersen, D., Nelson, M. A., WernerWashburne, M., Selitrennikoff, C. P., Kinsey, J. A., Braun, E. L., Zelter, A., Schulte, U., Kothe, G. O., Jedd, G., Mewes, W., Staben, C., Marcotte, E., Greenberg, D., Roy, A., Foley, K., Naylor, J., Stange-Thomann, N., Barrett, R., Gnerre, S., Kamal, M., Kamvysselis, M., Mauceli, E., Bielke, C., Rudd, S., Frishman, D., Krystofova, S., Rasmussen, C., Metzenberg, R. L., Perkins, D. D., Kroken, S., Cogoni, C., Macino, G., Catcheside, D., Li, W., Pratt, R. J., Osmani, S. A., DeSouza, C. P., Glass, L., Orbach, M. J., Berglund, J. A., Voelker, R., Yarden, O., Plamann, M., Seiler, S., Dunlap, J., Radford, A., Aramayo, R., Natvig, D. O., Alex, L. A., Mannhaupt, G., Ebbole, D. J., Freitag, M., Paulsen, I., Sachs, M. S., Lander, E. S., Nusbaum, C., and Birren, B. 2003. The genome sequence of the filamentous fungus Neurospora crassa. Nature 422:859-868.

20. Godoy, G., Steadman, J. R., Dickman, M. B., and Dam, R. 1990. Use of mutants to demonstrate the role of oxalic acid in pathogenicity of Sclerotinia sclerotiorum on Phaseolus vulgaris. Physiol. Mol. Plant Pathol. 37:179-191.

21. Gustin, M. C., Albertyn, J., Alexander, M., and Davenport, K. 1998. MAP kinase pathways in the yeast Saccharomyces cerevisiae. Microbiol. Mol. Biol. Rev. 62:1264-1300.
22. Hadar, Y., Henis, Y., and Chet, I. 1981. The potential for the formation of sclerotia in submerged mycelium of Sclerotinia rolfsii. J. Gen. Microbiol. 122:137-141.

23. Henis, Y., Chet, I., and Avizohar-Hershenzon, Z. 1965. Nutritional and mechanical factors involved in mycelium growth and production of sclerotia by Sclerotium rolfsii in artificial medium and amended soil. Phytopathology 55:87-91

24. Impey, S., Obrietan, K., Wong, T. S., Poser, S., Yano, Y., Wayman, G., Deloulme, J. C., Chan, G., and Storm, D. R. 1998. Cross talk between ERK and PKA Is required for $\mathrm{Ca}^{2+}$ stimulation of CREB-dependent transcription and ERK nuclear translocation. Neuron 21:869-883.

25. Jin, T., Amzel, M., Devreotes, P. N., and Wu, L. 1998. Selection of G $\beta$ subunits with point mutations that fail to activate specific signaling pathways in vivo: Dissecting cellular responses mediated by a heterotrimeric G protein in Dictyostelium discoideum. Mol. Biol. Cell 9:29492961.

26. Joham, H. E. 1943. A nutritional study of the fungus Sclerotium rolfsii. M.S. thesis. College Station, TX.

27. Jurick, W. M., II, Dickman, M. B., and Rollins, J. A. 2004. Characterization and functional analysis of a cAMP-dependent protein kinase A catalytic subunit gene (pkal) in Sclerotinia sclerotiorum. Physiol. Mol. Plant Pathol. 64:155-163.

28. Kaffarnik, F., Muller, P., Leibundgut, M., Kahmann, R., and Feldbrugge, M. 2003. PKA and MAPK phosphorylation of Prf1 allows promoter discrimination in Ustilago maydis. EMBO J. 22:5817-5826.

29. Kawasaki, H., Springett, G. M., Mochizuki, N., Toki, S., Nakaya, M., Matsuda, M., Housman, D. E., and Graybiel, A. M. 1998. A family of cAMP-binding proteins that directly activate Rap1. Science 282:22752279.

30. Kritzman, G., Chet, I., and Henis, Y. 1976. Metabolism of L-threonine and its relationship to sclerotium formation in Sclerotium rolfsii. J. Gen. Microbiol. 95:78-86.

31. Laidley, C. W., Cohen, E., and Casida, J. E. 1997. Protein phosphatase in neuroblastoma cells: $[3 \mathrm{H}]$ cantharidin binding site in relation to cytotoxicity. J. Pharmacol. Exp. Ther. 280:1152-1158.

32. Lee, N., D'Souza, C. A., and Kronstad, J. W. 2003. Of smuts, blasts, mildews, and blights: cAMP signaling in phytopathogenic fungi. Annu. Rev. Phytopathol. 41:399-427.

33. Lee, N., and Kronstad, J. W. 2002. ras 2 controls morphogenesis, pheromone response, and pathogenicity in the fungal pathogen Ustilago maydis. Eukaryotic Cell 1:954-966.

34. Lengeler, K. B., Davidson, R. C., D’Souza, C., Harashima, T., Shen, W. C., Wang, P., Pan, X., Waugh, M., and Heitman, J. 2000. Signal transduction cascades regulating fungal development and virulence. Microbiol. Mol. Biol. Rev. 64:746-785.

35. Martin, H., Castellanos, M. C., Cenamor, R., Sanchez, M., Molina, M., and Nombela, C. 1996. Molecular and functional characterization of a mutant allele of the mitogen-activated protein-kinase gene SLT2 (MPK1) rescued from yeast autolytic mutants. Curr. Genet. 29:516-522.

36. Martin, H., Rodriguez-Pachon, J. M., Ruiz, C., Nombela, C., and Molina, M. 2000. Regulatory mechanisms for modulation of signaling through the cell integrity Slt2-mediated pathway in Saccharomyces cerevisiae. J. Biol. Chem. 275:1511-1519.

37. Maxwell, D. P., and Lumsden, R. D. 1970. Oxalic acid production by Sclerotinia sclerotiorum in infected bean and in culture. Phytopathology 60:1395-1398.

38. Mitchell, T. K., and Dean, R. A. 1995. The cAMP-dependent protein kinase catalytic subunit is required for appressorium formation and pathogenesis by the rice blast pathogen Magnaporthe grisea. Plant Cell 7:18691879.

39. Nelson, D. L., and Cox, M. M. 2000. Lehninger Principles of Biochemistry, 3rd ed. Worth Publishers, New York.

40. Okon, Y., Chet, I., and Henis, Y. 1972. Lactose-induced synchronous sclerotium formation in Sclerotium rolfsii and its inhibition by ethanol. J. Gen. Microbiol. 71:465-470.

41. Purdy, L. H. 1979. Sclerotinia sclerotiorum: History, diseases and symptomatology, host range, geographic distribution and impact. Phytopathology 69:875-880.

42. Robinson, M., Riov, J., and Sharon, A. 1998. Indole-3-acetic acid biosynthesis in Colletotrichum gloeosporioides f. sp. aeschynomene. Appl. Environ. Microbiol. 64:5030-5032.

43. Rollins, J. A., and Dickman, M. B. 1998. Increase in endogenous and exogenous cyclic AMP levels inhibits development in Sclerotinia sclerotiorum. Appl. Environ. Microbiol. 64:2539-2544.

44. Rollins, J. A., and Dickman, M. B. 2001. pH signaling in Sclerotinia sclerotiorum: Identification of a pacC/RIM1 homolog. Appl. Environ. Microbiol. 67:75-81.

45. Rupp, S. E., Summers, H. L., Madhani, H., and Fink, G. 1999. MAP kinase and cAMP filamentation signaling pathways converge on the unusually large promoter of the yeast FLO11 gene. EMBO J. 18:1257-1269. 
46. Sambrook, J., Fritsch, E. F., and Maniatis, T. 1989. Molecular Cloning: A Laboratory Manual. Cold Spring Harbor Laboratory, Cold Spring Harbor, NY.

47. Steadman, J. R. 1979. Control of plant diseases caused by Sclerotinia species. Phytopathology 69:904-907.

48. Suzuki, N., Choe, H. R., Nishida, Y., Yamawaki-Kataoka, Y., Ohnishi, S., Tamaoki, T., and Kataoka, T. 1990. Leucine-rich repeats and carboxyl terminus are required for interaction of yeast adenylate cyclase with RAS proteins. Proc. Natl. Acad. Sci. USA 87:8711-8715.

49. Tourneau, D. L. 1979. Morphology, cytology, and physiology of Sclerotinia species in culture. Phytopathology 69:887-890.

50. Townsend, B. B., and Willetts, H. J. 1954. The development of sclerotia of certain fungi. Ann. Bot. 21:153-166.

51. Tu, J. C. 1997. An integrated control of white mold (Sclerotinia sclerotiorum) of beans, with emphasis on recent advances in biological control. Bot. Bull. Acad. Sin. 38:73-76.

52. Willetts, H. J. 1997. Morphology, development and evolution of stromata/ sclerotia and macroconidia of the Sclerotiniaceae. Mycol. Res. 101:939-52.

53. Willetts, H. J., and Bullock, S. 1992. Developmental biology of sclerotia. Mycol. Res. 96:801-816.

54. Xu, J. R. 2000. MAP kinases in fungal pathogens. Fungal Genet. Biol. 31:137-152.
55. Xu, J. R., and Hamer, J. E. 1996. MAP kinase and cAMP signaling regulate infection structure formation and pathogenic growth in the rice blast fungus Magnaporthe grisea. Genes Dev. 10:26962706.

56. Xu, J. R., Urban, M., Sweigard, J. A., and Hammer, J. E. 1997. The $C P K A$ gene of Magnaporthe grisea is essential for appressorial penetration. Mol. Plant-Microbe Interact. 10:187-194.

57. Yamaguchi, T., Nagao, S., Wallace, D. P., Belibi, F. A., Cowley, B. D. Pelling, J. C., and Grantham, J. J. 2003. Cyclic AMP activates B-Raf and ERK in cyst epithelial cells from autosomal-dominant polycystic kidneys. Kidney Int. 63:1983-1994.

58. Yang, Z., and Dickman, M. B. 1999. Colletotrichum trifolii mutants disrupted in the catalytic subunit of cAMP-dependent protein kinase are non-pathogenic. Mol. Plant-Microbe Interact. 12:430-439.

59. Yang, Z., and Dickman, M. B. 1999. Molecular cloning and characterization of Ct-PKAR, a gene encoding the regulatory subunit of cAMPdependent protein kinase in Colletotrichum trifolii. Arch. Microbiol. 171:249-256.

60. Zhang, Y., Lamm, R., Pillonel, C., Lam, S., and Xu, J. R. 2002. Osmoregulation and fungicide resistance: The Neurospora crassa os-2 gene encodes a HOG1 mitogen-activated protein kinase homologue. Appl. Environ. Microbiol. 68:532-538. 